\title{
Effect of cholesterol lowering treatment on positive exercise tests in patients with hypercholesterolaemia and normal coronary angiograms
}

\author{
A P Mansur, C V Serrano Jr, J C Nicolau, L A M César, J A F Ramires
}

\begin{abstract}
Aim-To assess the impact of cholesterol lowering on positive exercise stress tests in hypercholesterolaemic patients with normal coronary angiograms.

Methods-43 non-diabetic patients aged 43-61 years, with total serum cholesterol concentrations of more than $7.75 \mathrm{mmol} / 1$, positive exercise tests, and normal coronary angiograms, were started on the American Heart Association step 1 diet. After 12 weeks these patients were randomly assigned to treatment for another 16 weeks with the diet alone (diet group, $n=20$ ) or with the diet plus lovastatin or simvastatin (statin group, $n=23$ ). After this 28 week run in period, statins were withdrawn and lipid profile tests and exercise tests were done and repeated 20 weeks later.

Results-At week 28, the statin group but not the diet group had significant reductions from baseline (week 12) in plasma total cholesterol $(p<0.0001)$, low density lipoprotein $(p<0.0001)$, and triglyceride $(p<0.0001)$. The number of patients with positive exercise tests decreased from 23 to three in the statin group and from 20 to 15 in the diet group $(p=0.01)$. After the final 20 weeks without statins, lipid profiles returned to baseline levels in all 17 patients remaining in the statin group, and exercise tests were again positive in $\mathbf{1 5}$ of these patients.

Conclusions-In hypercholesterolaemic patients with normal coronary arteries, cholesterol lowering treatment reduces myocardial ischaemia, as shown by the beneficial effects on exercise testing. (Heart 1999;82:689-693)
\end{abstract}

Heart Institute, São Paulo University, Av Dr Enéas de Carvalho Aguiar 44, 05403-000 São Paulo, Brazil A P Mansur C V Serrano J C Nicolau L A M César J A F Ramires

Correspondence to: Dr Mansur. email: corantonio@ incor.usp.br

Accepted for publication 13 April 1999
Keywords: hypercholesterolaemia; exercise stress testing; myocardial ischaemia; endothelium

Hypercholesterolaemia advances the atherosclerotic process by increasing low density lipoprotein (LDL) cholesterol concentration and accumulation, enhancing endothelial permeability to LDL cholesterol, and promoting cholesterol modification. ${ }^{12}$ Modified cholesterol (for example, oxidised LDL) causes endothelial dysfunction, ${ }^{3}$ which in experimental models appears to precede even the earliest structural endothelial alteration related to the atherosclerotic process. ${ }^{4}$ Effectively lowering plasma total cholesterol and LDL cholesterol in hypercholesterolaemic patients with and without coronary artery disease improves the endothelium dependent vasomotor response to acetylcholine. ${ }^{56}$ Endothelial function also improves within two to four weeks after starting cholesterol lowering treatment. $^{78}$ There is evidence that lowering cholesterol is associated with modest but measurable regression of new atherosclerotic lesions. ${ }^{9}$ In large scale therapeutic trials, the vascular benefits of cholesterol lowering treatment have been reflected in a substantial reduction in the need for coronary revascularisation procedures. ${ }^{10}{ }^{11}$ Recent trials have shown that effective lipid lowering treatment in patients with obstructive coronary artery disease improves measures of transient myocardial ischaemia on positron emission tomography and ambulatory ECG monitoring. ${ }^{12} 13$

Whether or not reducing cholesterol levels improves myocardial ischaemia, however, has not been well defined in the clinical setting. In this randomised controlled trial, we assessed the effect of cholesterol lowering treatment on myocardial ischaemia as measured by ST segment depression during exercise stress test in patients with hypercholesterolaemia and normal coronary angiograms.

\section{Methods}

SUBJECTS

Patients were eligible for the study if they had a total serum cholesterol concentration greater than $7.75 \mathrm{mmol} / \mathrm{l}(300 \mathrm{mg} / \mathrm{dl})$, a positive exercise stress test (characterised by ST segment depression), were not receiving cholesterol lowering drugs, and had normal coronary angiograms and left ventricular function. All patients had typical or atypical angina or were asymptomatic but had a positive exercise test. Patients with diabetes, a diastolic blood pressure of more than $95 \mathrm{~mm} \mathrm{Hg}$, or any evidence of cardiovascular disease were excluded. Other exclusion criteria were basal electrocardiographic abnormalities that might limit the interpretation of the exercise testing, such as left ventricular hypertrophy with ST/T wave changes, left bundle branch block, pre-excitation syndrome, and intraventricular conduction disturbance. Patients with previous hypersensitivity to statins were also excluded.

The study protocol was approved by the institutional ethics committee, the procedures followed were in accordance with institutional guidelines, and written informed consent was obtained from the patients. 
Table 1 Prestudy patient characteristics

\begin{tabular}{lccc}
\hline & Diet $(n=20)$ & Statin $(n=23)$ & p Value \\
\hline Age (years) & $52(6)$ & $50(4)$ & 0.200 \\
Male/female (n) & $17 / 3$ & $18 / 5$ & 0.862 \\
Family history of CAD (n (\%)) & $5(25)$ & $6(26)$ & 0.783 \\
Smoking (n (\%)) & $3(15)$ & $3(13)$ & 0.801 \\
Asymptomatic (n (\%)) & $15(75)$ & $13(57)$ & 0.360 \\
Angina (n (\%)) & & $5(22)$ & \\
$\quad$ Typical & $2(10)$ & $5(22)$ & 0.518 \\
$\quad$ Atypical & $3(15)$ & $9.46(1.27)$ & 0.845 \\
Total cholesterol (mmol/1) & $9.59(0.85)$ & $7.81(0.72)$ & 0.898 \\
LDL cholesterol (mmol/1) & $7.84(0.80)$ & $1.19(0.39)$ & 0.555 \\
HDL cholesterol (mmol/1) & $1.11(0.49)$ & $2.48(0.65)$ & 0.919 \\
Triglyceride (mmol/1) & $2.46(0.62)$ & &
\end{tabular}

Values are mean (SD) unless stated otherwise.

${ }^{\star} \mathrm{p}$ Values for age, total cholesterol, LDL cholesterol, HDL cholesterol, and triglyceride are from a Student $t$ test; for sex from a $\chi^{2}$ test; and for asymptomatic and smoking from comparison of two proportions.

HDL, high density lipoprotein; LDL, low density lipoprotein.

EXERCISE TESTING AND CORONARY ANGIOGRAPHY Exercise stress testing was performed according to the Bruce protocol under continuous 12 lead ECG monitoring. ${ }^{14}$ Blood pressure and heart rate were measured throughout the test or when clinically indicated. Exercise testing was terminated upon physical exhaustion, onset of angina, or when ST segment depression was $3 \mathrm{~mm}$ or more. Exercise testing was considered positive when significant horizontal or down sloping ST segment depression greater than $1 \mathrm{~mm}$ (for men) and $2 \mathrm{~mm}$ (for women) occurred at 0.08 seconds after the J point.

After exercise testing, all patients underwent cardiac catheterisation and coronary angiography using the Sones and Shirey method. ${ }^{15}$

PROTOCOL DESIGN

Once patients met the inclusion criteria, they received dietary instructions from a registered dietician and began the American Heart Association step 1 diet, ${ }^{16}$ which they continued for 12 weeks (washout period and study weeks $0-11)$. The patients were then randomly assigned to 16 weeks of treatment (run in period and study weeks 12-28) either on the American Heart Association step 1 diet alone (diet group) or on the same diet plus lovastatin or simvastatin (statin group). Follow up visits, serial lipid profiles, and exercise tests were scheduled at the start and then every four weeks during the run in period (at study weeks $12,16,20,24$, and 28). The initial daily dose of lovastatin $(20 \mathrm{mg})$ or simvastatin $(10 \mathrm{mg})$ was adjusted during the study to achieve total cholesterol concentrations below $6.21 \mathrm{mmol} / 1$ (240 $\mathrm{mg} / \mathrm{dl})$. Thereafter, statins were withdrawn and both groups remained on the step 1 diet only for 20 additional weeks (post-statin period, study weeks 29-48). Lipid profiles and exercise tests were repeated at the end of week 48 .

\section{LABORATORY MEASUREMENTS}

Plasma lipid concentrations were obtained after a 12 hour overnight fasting period. Total cholesterol (TC) and triglyceride (TG) concentrations were determined by standard enzymatic methods, ${ }^{17}$ high density lipoprotein (HDL) concentrations by selective precipitation with dextran-magnesium chloride, ${ }^{18}$ and LDL concentrations by the Freidewald formula: $\mathrm{LDL}=\mathrm{TC}-[\mathrm{HDL}+(\mathrm{TG} / 2.19)] .{ }^{19}$

\section{EFFICACY AND SAFETY MEASUREMENTS}

During the follow up visits, patients had a complete medical examination, an ECG, and routine blood analyses. They were also asked if they had experienced any symptoms that could have been related to statin treatment. We determined whether the patients had had any intercurrent illness or complication requiring interruption of statin treatment. Compliance was verified by monitoring the amount of drug dispensed.

\section{STATISTICAL ANALYSIS}

Age and total, HDL, and LDL cholesterol were analysed using Student's $t$ test for comparisons between groups. One way analysis of variance was used for comparisons within groups for continuous variables such as triglyceride, total, HDL, VLDL, and LDL cholesterol, heart rate, and systolic and diastolic blood pressure. The $\chi^{2}$ statistic, or Fischer's exact test when appropriate, was used to analyse categorical variables (sex, symptoms, exercise stress test). A p value of $<0.05$ was considered statistically significant. Data are expressed as mean (SD) unless otherwise specified.

\section{Results}

PATIENT CHARACTERISTICS

Forty three consecutive patients were enrolled in the study: 35 men and eight women, aged 43 to 61 years (mean (SD), 51 (8) years). Twenty patients were randomly assigned to remain on

Table 2 Mean plasma lipid concentrations for the diet group (D) and the statin group (S) during the run in period (week 28) and post-statin period (week 48)

\begin{tabular}{|c|c|c|c|c|c|c|c|}
\hline & & \multicolumn{6}{|l|}{ Week } \\
\hline & & 12 & 16 & 20 & 24 & 28 & 48 \\
\hline \multirow[t]{2}{*}{ Total cholesterol (mmol/l) } & $\mathrm{D}$ & $9.59(0.85)$ & $9.52(1.06)$ & $9.70(1.09)$ & $9.49(1.20)$ & $9.44(1.27)$ & $9.34(1.14)$ \\
\hline & $\mathrm{S}$ & $9.46(1.16)$ & $6.90(1.34)^{\star \star \star \star}$ & $6.36(1.50)^{\star \star \star \star}$ & $5.72(0.78)^{\star \star \star}$ & $5.79(0.75) \dagger^{\star \star \star}$ & $9.13(0.83) \ddagger$ \\
\hline \multirow{2}{*}{ LDL cholesterol $(\mathrm{mmol} / \mathrm{l})$} & $\mathrm{D}$ & $7.84(0.80)$ & $7.86(0.91)$ & $7.76(0.98)$ & $7.89(0.83)$ & $7.86(0.91)$ & $7.78(0.98)$ \\
\hline & $\mathrm{S}$ & $7.81(0.72)$ & $4.73(0.54)^{\star \star \star}$ & $4.53(0.59)^{\star \star \star}$ & $4.14(0.52)^{\star \star \star}$ & $4.19(0.49) \dagger^{\star \star \star}$ & $7.73(0.91) \ddagger$ \\
\hline \multirow[t]{2}{*}{ HDL cholesterol ( $\mathrm{mmol} / \mathrm{l})$} & $\mathrm{D}$ & $1.11(0.49)$ & $1.16(0.39)$ & $1.14(0.39)$ & $1.11(0.41)$ & $1.16(0.47)$ & $1.03(0.34)$ \\
\hline & $\mathrm{S}$ & $1.19(0.39)$ & $1.32(0.52)$ & $1.29(0.49)$ & $1.29(0.47)$ & $1.32(0.54)$ & $0.98(0.23) \ddagger$ \\
\hline \multirow[t]{2}{*}{ VLDL cholesterol (mmol/l) } & $\mathrm{D}$ & $0.65(0.31)$ & $0.49(0.31)$ & $0.52(0.23)$ & $0.47(0.26)$ & $0.44(0.16) \dagger$ & $0.49(0.47)$ \\
\hline & $\mathrm{S}$ & $0.49(0.18)^{\star}$ & $0.85(0.047)^{\star \star}$ & $0.54(0.18)$ & $0.28(0.13)^{\star \star}$ & $0.34(0.21)$ & $0.44(0.39)$ \\
\hline \multirow[t]{2}{*}{ Triglyceride $(\mathrm{mmol} / \mathrm{l})$} & $\mathrm{D}$ & $2.46(0.62)$ & $2.53(0.59)$ & $2.28(0.49)$ & $2.35(0.65)$ & $2.17(0.59)$ & $2.48(0.49)$ \\
\hline & $S$ & $2.48(0.65)$ & $3.90(1.06)^{\star \star \star}$ & $3.54(0.62)^{\star \star \star}$ & $2.07(0.57)$ & $2.09(0.57) \dagger$ & $2.22(0.47)$ \\
\hline
\end{tabular}

Values are mean (SD).

$t \mathrm{p}<0.001$, within group values for triglyceride, total cholesterol, and cholesterol fraction from week 12 to week 28 changes, by analysis of variance. $\ddagger \mathrm{p}<0.001$ values from week 28 to week 48, by Student's $t$ test.

Significant same week, between group lipid comparisons using Student's $t$ test are given by ${ }^{\star}$ for $\mathrm{p}<0.05,{ }^{\star \star}$ for $\mathrm{p}<0.01$, and ${ }^{\star \star \star}$ for $\mathrm{p}<0.001$. 
Table 3 Exercise test variables and per cent positive tests for the diet group and the statin group during the run in period (week 28) and the post-statin period (week 48)

\begin{tabular}{|c|c|c|c|c|c|c|c|}
\hline & & \multicolumn{6}{|l|}{ Week } \\
\hline & & 12 & 16 & 20 & 24 & 28 & 48 \\
\hline \multirow[t]{2}{*}{$\mathrm{SBP}(\mathrm{mm} \mathrm{Hg})$} & $\mathrm{D}$ & $181(8)$ & $184(7)$ & $183(8)$ & $185(6)$ & $184(8)$ & $183(7)$ \\
\hline & $S$ & $188(13)$ & $183(10)$ & $184(7)$ & $183(5)$ & $182(8)$ & $184(9)$ \\
\hline \multirow{2}{*}{$\mathrm{DBP}(\mathrm{mm} \mathrm{Hg})$} & $\mathrm{D}$ & $82(3)$ & $80(5)$ & $80(3)$ & $80(6)$ & $79(4)$ & $80(5)$ \\
\hline & $S$ & $85(5)$ & $82(7)$ & $78(5)$ & $79(4)$ & $80(5) \dagger$ & $83(6)$ \\
\hline \multirow[t]{2}{*}{ HR (beats/min) } & $\mathrm{D}$ & $147(14)$ & $145(10)$ & $148(11)$ & $147(8)$ & $146(9)$ & $147(10)$ \\
\hline & $S$ & $144(11)$ & $143(12)$ & $148(9)$ & $154(11)^{\star}$ & $157(5) \dagger^{\star \star \star}$ & $153(8) \ddagger^{\star}$ \\
\hline \multirow[t]{2}{*}{$\mathrm{EST}+(\%)$} & $\mathrm{D}$ & $20(100)$ & $18(90)$ & $17(85)$ & $15(75)$ & $15(75)$ & $14(93.3)$ \\
\hline & $\mathrm{S}$ & $23(100)$ & $18(78.2)$ & $11(47.8)$ & $5(21.7)$ & $3(13)$ & $15(88.2)$ \\
\hline
\end{tabular}

Values are mean (SD) or mean (\%).

$t \mathrm{p}<0.0001$, within group comparison of change from week 12 to week 28 by one way analysis of variance; $\neq \mathrm{p}=0.048$, within group comparison of change from week 28 to week 48 by Student's $t$ test. Significant same week, between group comparisons using Student's $t$ test are given by ${ }^{\star}$ for $\mathrm{p}<0.05$ and ${ }^{\star \star \star}$ for $\mathrm{p}<0.001$

$\mathrm{Sp}=0.01$ and $\Psi \mathrm{p}=0.029$, between group comparison of EST + at week $12 v$ week 28 and week $28 v$ week 48, respectively, using Fischer's exact test.

$\mathrm{D}$, diet group; DBP, diastolic blood pressure; EST + , positive exercise stress test; HR, heart rate; S, statin group; SBP, systolic blood pressure.

step 1 diet (the diet group) and 23 to treatment with lovastatin or simvastatin as well as diet (the statin group). The prestudy characteristics of the patients are shown in table 1 .

CLINICAL FOLLOW UP

All patients completed the 12 week wash out and 16 week run in periods. Fifteen of the 20 patients $(75 \%)$ in the diet group and 17 of the $23(74 \%)$ in the statin group completed the 20 week post-statin period. Five patients in the diet group $(25 \%)$ and six in the statin group $(26 \%)$ were lost to follow up after the run in period. No cardiovascular events and no adverse effects attributable to the study drugs occurred during the study period. In the statin group, the mean daily dose was 55.1 (18.8) mg of lovastatin or $13.6(18.8) \mathrm{mg}$ of simvastatin.

CHANGES IN LIPID PROFILES

Follow up plasma lipid levels were tested at intervals of four weeks from the end of week 12 through to the end of week 28 (table 2). At the end of the 16 week period of statin treatment there were marked reductions from the week 12 baseline in the statin group, but not in the diet group, in plasma concentrations of total cholesterol (from $9.46(1.26)$ to $5.79(0.75)$ $\mathrm{mmol} / \mathrm{l} ; \mathrm{p}<0.0001)$, LDL cholesterol (from

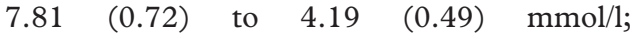
$\mathrm{p}<0.0001$ ), and triglyceride (from $2.48(0.65)$ to $2.09(0.57) \mathrm{mmol} / \mathrm{l} ; \mathrm{p}<0.0001)$. The greatest reductions in total cholesterol and LDL cholesterol occurred within the first four weeks of the run in period (by study week 16), after which only slight and gradual lowering was observed. Lipid profiles in the diet group were unchanged throughout the run in period. HDL cholesterol concentrations remained unchanged in both groups throughout the wash out and run in periods.

After 20 weeks without statins (post-statin period), total cholesterol and LDL cholesterol levels returned to baseline in all 15 patients who remained in the diet group and all 17 still in the statin group. In the statin group, HDL cholesterol decreased from $1.32(0.54) \mathrm{mmol} / 1$ at week 28 to $0.98(0.23) \mathrm{mmol} / \mathrm{l}$ at week 48 $(\mathrm{p}=0.009 ;$ table 3$)$.
EXERCISE STRESS TEST FOLLOW UP

At the end of week 16 of the run in period, there was a marked reduction in the number of positive exercise tests in the statin group as compared with the diet group (75\% s 13\%; $\mathrm{p}=0.01$; table 2 ). Normalisation of exercise stress test results occurred gradually throughout the run in period. The statin group, but not the diet group, also had significantly reduced diastolic blood pressure and heart rate compared with baseline (both $\mathrm{p}<0.0001$ ) during exercise testing at the end of the run in period (table 3).

After 20 weeks without statins, 14 of 15 patients $(93 \%)$ in the diet group still had positive exercise test results and 15 of 17 patients $(88 \%)$ in the statin group again had positive exercise tests (table 3 ). In the statin group, heart rate decreased from 157 (5) beats/min at week 28 to 153 (8) beats $/ \mathrm{min}$ at week 48 ( $p=0.048$; table 3 ). Diastolic blood pressure was unchanged throughout the post-statin period.

\section{Discussion}

This randomised, controlled study showed that myocardial ischaemia in hypercholesterolaemic patients with normal coronary arteries, as indicated by ST segment depression during exercise stress tests, can be ameliorated by cholesterol lowering drug treatment. Statin treatment reduced the number of patients with positive exercise test results and improved exercise endurance (higher maximum heart rate and lower maximum diastolic blood pressure). In contrast, diet alone produced little change in cholesterol and did not improve myocardial ischaemia induced by exercise testing. The beneficial effect of lowering plasma cholesterol concentrations by statin treatment may be related to normalisation of coronary endothelial function.

Research has shown that coronary risk factors, such as increased plasma LDL cholesterol $^{20}{ }^{21}$ and oxidised LDL, ${ }^{22}$ adversely affect endothelial vasomotor responses in humans. Intracoronary infusion of acetylcholine has been shown to dilate normal human coronary arteries by inducing the release of 
nitric oxide (endothelium derived relaxing factor, EDRF). ${ }^{23}$ Ludmer et al observed a vasodilator response in normal coronary arteries after acetylcholine infusion, but a paradoxical constriction in atherosclerotic arteries reflecting the loss of EDRF. ${ }^{24}$ Experimental data suggest that dyslipidaemia induced endothelial dysfunction may in part be the result of nitric oxide inactivation, probably caused by the increased vascular production of oxygen derived free radicals ("oxidative stress") that occurs in hypercholesterolaemic states. ${ }^{25}$

The vasomotor responses of epicardial arteries during exercise also appear to be modulated by EDRF. ${ }^{26}$ Normal epicardial arteries dilate during exercise, while atherosclerotic arteries constrict. Similar responses occur when the stimulus is an increase in heart rate.$^{27}$ Exercise is associated with increased coronary blood flow and activation of the sympathetic system. In human atherosclerotic coronary arteries, the loss of flow mediated ${ }^{28}$ and $\alpha_{2}$ adrenergic stimulated EDRF release ${ }^{29}$ may contribute to abnormal coronary vasoconstriction and thus to myocardial ischaemia. ${ }^{30} 31$

Heart rate and systolic and diastolic blood pressure, among other factors, determine myocardial oxygen consumption. The only significant mechanism available to the heart to increase its oxygen consumption is to increase perfusion, and there is a direct linear relation between myocardial oxygen consumption and coronary blood flow in normal individuals. The principal mechanism for increasing coronary blood flow during exercise is decreased resistance at the coronary arteriolar level. Dynamic changes in vascular tone in an atherosclerotic coronary artery may result in diminished coronary flow during dynamic exercise. Thus regional left ventricular myocardial ischaemia may result not only from an increase in myocardial oxygen demand during exercise but also from a limitation of coronary flow as a result of coronary vasoconstriction in hypercholesterolaemic conditions. ${ }^{27}$

The anti-ischaemic action of 3-hydroxy-3methylglutaryl coenzyme A reductase (HMGCoA) inhibitors during exercise has not been reported before. In this study, aggressive lipid lowering for four months reduced the incidence of myocardial ischaemia. The effect of statin treatment on endothelial function might in part explain our findings. Endothelial vasomotor dysfunction limits the coronary flow reserve and predisposes to myocardial ischaemia at two points in the atherosclerotic artery: it contributes to epicardial vasoconstriction, and it leads to an increase in the tone of resistance vessels so that myocardial blood flow is unable to meet an increase in metabolic demand. ${ }^{32}$ Disturbances in coronary endothelial function associated with hypercholesterolaemia may be reversible at both points if adequately treated.

This study complements other research indicating that lipid lowering treatment reduces the incidence of myocardial ischaemia and improves endothelial function. Andrews et $a l,{ }^{12}$ using ambulatory ECG monitoring, showed a $65 \%$ reduction in myocardial ischae- mia during daily life activities after lovastatin treatment in patients with coronary heart disease. Gould et al used positron emission tomography to demonstrate the beneficial effect of lipid lowering on the coronary microcirculation. ${ }^{13}$ In our study, lipid lowering reduced the incidence of myocardial ischaemia at maximal exercise, an effect that may be mediated by normalisation of coronary endothelial function.

The absence of an ergonovine induced spasm provocation test during coronary angiography and nuclear myocardial scintigraphy are important limitations of this study. Although generally safe, irreversible occlusion may occur with the ergonovine provocation test.

This study was supported in part by research grants from the Fundação E J Zerbini, São Paulo, Brazil

1 Vogel RA. Coronary risk factors, endothelial function, and atherosclerosis: a review. Clin Cardiol 1997;20:426-32.

2 Glasser SP, Selwyn AP, Ganz P. Atherosclerosis: risk factors and the vascular endothelium. Am Heart F 1996;131:37984.

3 Simon BC, Cunningham LD, Cohen RA. Oxidized low density lipoproteins cause contraction and inhibit endothelium-dependent relaxation in the pig coronary artery. F Clin Invest 1990;86:75-9.

4 Mano T, Masuyama T, Yamamoto K, et al. Endothelial dysfunction in the early stage of atherosclerosis precedes appearance of intimal lesions assessable with intravascular ultrasound. Am Heart f 1996;131:231-8.

5 Leung WH, Lau CP, Wong CK. Beneficial effect of cholesterol-lowering therapy on coronary endotheliumdependent relaxation in hypercholesterolemic patients. Lancet 1993;341:1496-500.

6 Treasure CB, Klein JL, Weintraub WS, et al. Beneficial effects of cholesterol-lowering therapy on the coronary endothelium in patients with coronary artery disease. $N$ Engl f Med 1995;332:481-7.

7 Stroes ESG, Koomans HÁ, de Bruin TWA, et al. Vascular function in the forearm of hypercholesterolaemic patients off and on lipid-lowering medication. Lancet 1995;346: $467-71$

8 Vogel RA, Corretti MC, Plotnick GD. Changes in flow-mediated brachial artery vasoactivity with lowering of
desirable cholesterol levels in healthy middle-aged men. Am $\mathcal{F}$ Cardiol 1996;77:37-40.

9 Selwyn AP, Kinlay S, Libby P, et al. Atherogenic lipids, vascular dysfunction, and clinical signs of ischemic heart disease. Circulation 1997;95:5-7.

10 Randomized trial of cholesterol lowering therapy in 4444 patients with coronary heart disease: the Scandinavian

11 Shepherd J, Cobbe SM, Ford I, et al. Prevention of coronary heart disease with pravastatin in men with hypercholesterolemia: West of Scotland Coronary Prevenion Study Group. N Engl F Med 1995;333:1301-7.

12 Andrews TC, Raby K, Barry J, et al. Effect of LDL cholesterol reduction on myocardial ischemia in patients with coronary disease. Circulation 1997;95:324-8.

13 Gould KL, Martucci JP, Goldberg DI, et al. Short-term cholesterol lowering decreases size and severity of perfusion abnormalities by positron emission tomography after dipyridamole in patients with coronary artery disease: a potential noninvasive marker of healing coronary endothelium. Circulation 1994;89:1530-8.

14 Froelicher VF. Exercise and the heart. Clinical concepts, 2nd ed. Chicago: Year Book Medical Publishers, 1987.

5 Sones FM, Shirey EK. Cinecoronary arteriography. Mod Concepts Cardiovasc Dis 1962;31:735-7.

16 Report of the National Cholesterol Education Program expert panel on detection, evaluation, and treatment of 36-69

17 Röschlau P, Bernt E, Gruber W. Enzymatische Bestimmung des Gesamt-cholesterins im Serum. Klin Chem Klin Biochem 1974;12:403-7.

18 Bucolo G, David H. Quantitative determination of serum triglycerides by the use of enzymes. Clin Chem 1973;19: 476-82.

19 Warnick GR, Benderson JM, Albers JJ. Dextran sulfate precipitation procedure for quantification of high-density lipoprotein. Clin Chem 1982;28:1379-88.

20 Freidewald WT, Levy RI, Fredrickson DS. Estimation of the concentration of low-density lipoprotein cholesterol in plasma, without use of the preparative ultracentrifuge. Clin Chem 1972;18:499-502.

21 Egashira K, Inou T, Hirooka Y, et al. Impaired coronary blood flow response to acetylcholine in patients with coronary risk factors and proximal atherosclerotic lesions. $\mathcal{F}$ Clin Invest 1993;91:29-37.

22 Zeiher AM, Drexler H, Wollschläger $\mathrm{H}$, et al. Modulation of coronary vasomotor tone in humans: progressive endothe- 
lial dysfunction with different early stages of coronary atherosclerosis. Circulation 1991;83:391-401.

23 Anderson TJ, Meredith IT, Yeung AC, et al. Endotheliumdependent coronary vasomotion relates to the susceptibility of LDL to oxidation in humans. Circulation 1996;93: ity of LDL $1647-50$.

24 Quyyumi AA, Dakak N, Andrews NP. Nitric oxide activity in the human coronary circulation: impact of risk factor for coronary atherosclerosis. F Clin Invest 1995;95:174755.

25 Ludmer PL, Selwyn AP, Shook TL, et al. Paradoxical vasoconstriction induced by acetylcholine in atherosclerotic coronary arteries. N Engl f Med 1986;315:1046-51.

26 Ohara Y, Peterson TE, Harrison DG. Hypercholesterolemia increases endothelial superoxide anion production. $7 \mathrm{Clin}$ Invest 1993;91:2546-51.

27 Gordon JB, Ganz P, Nabel EG, et al. Atherosclerosis and endothelial function influence the coronary vasomotor endothelial function influence the coronary vasom
response to exercise. F Clin Invest 1989;83:1946-52.
28 Nabel EG, Selwyn AP, Ganz P. Paradoxical narrowing of atherosclerotic coronary arteries induced by increases in heart rate. Circulation 1990;81:850-9.

29 Nabel EG, Selwyn AP, Ganz P. Large coronary arteries in humans are responsive to changes in blood flow: an endothelium-dependent mechanism that fails in patient with atherosclerosis. F Am Coll Cardiol 1990;16:349-56.

30 Vita JA, Treasure CB, Yeung AC. Patients with evidence of coronary endothelial dysfunction as assessed by acetylcholine infusion demonstrate marked increase in sensitivity to constrictor effects of catecholamines. Circulation 1992;85: 1390-7.

31 Seiler C, Suter TM, Hess OM. Exercise-induced vasomotion of angiographically normal and stenotic coronary arteries improves after cholesterol-lowering drug therapy with bezafibrate. 7 Am Coll Cardiol 1995;26:1615-22.

32 Andrews TC, Barry J, Naimi C, et al. The effect of LDL cholesterol reduction on myocardial ischemia in patients with coronary disease. Circulation 1997;95:324-8. 